Manuscript received November 1 , 2018; accepted for publication February 7, 2019; published online April 5, 2019.

1 Department of Civil and Environmental Engineering, Clarkson University, 8 Clarkson Ave., Potsdam, NY 13699, USA (Corresponding author), e-mail: rthomas@clarkson.edu, (1) https://orcid.org/0000-00027591-2405

2 Department of Civil and Environmental Engineering, Utah State University, 4110 Old Main HI., Logan, UT 84322, USA
Robert J. Thomas, ${ }^{1}$ Andrew D. Sorensen, ${ }^{2}$ and Marc Maguire ${ }^{2}$

\section{Analytical Models for 3-D Diffusion of Ions from Salt-Contaminated Aggregates}

\section{Reference}

R. J. Thomas, A. D. Sorensen, and M. Maguire, "Analytical Models for 3-D Diffusion of lons from Salt-Contaminated Aggregates," Advances in Civil Engineering Materials 8, no. 2 (2019): 1-19. https://doi.org/10.1520/ACEM20180137

\section{ABSTRACT}

This article presents an analytical investigation of the mobility of salts from contaminated aggregates in concrete. Salt-contaminated aggregates may have varied effects on the mechanical properties and durability of concrete. These depend primarily on the mobility of salts within the concrete matrix. Existing diffusion-based models for the mobility of salts in concrete focus on their intrusion from external sources (e.g., chloride penetration from deicing salts and brines). Such problems are well described by the closed-form solution of Fick's law for diffusion in one dimension from a continuous source. Salt-contaminated aggregates represent a case of diffusion from a finite internal source rather than intrusion from a continuous external source. When the source is internal-as in the case of salt-contaminated aggregates-diffusion occurs in three dimensions and the source is finite. The 1-D solution is ill conditioned to model this problem, so new diffusion models must be introduced. This article presents two models of varying complexity that are well conditioned to model diffusion of salts from contaminated aggregates. First, the problem is modeled using Fick's law for the simplified case of 3-D diffusion from an instantaneous point source in an infinite medium. Contaminated aggregates are treated as infinitesimal slugs of diffusing material. The same problem is then modeled using Fick's law for the more realistic case of 3-D diffusion from an instantaneous spherical source, where contaminated aggregates are treated as approximately spherical. The intent of this article is to present an academic discussion on how chloride ions might migrate within the concrete matrix if they are assumed to do so by diffusion alone.

\section{Keywords}

diffusion, 3-D, salt, chloride, aggregate, contaminated 


\section{Introduction}

Chloride-induced corrosion in steel-reinforced concrete is a leading cause of infrastructure deterioration. ${ }^{1}$ This phenomenon simultaneously results in section loss in the steel reinforcement ${ }^{2-4}$ and formation of expansive corrosion products. ${ }^{3,5-8}$ The former mechanism immediately reduces structural capacity, while the latter exerts cracking pressure on the concrete cover. The resulting cracks destroy the protection provided by the concrete cover and render the structure more vulnerable to chloride intrusion and damage from freezing and thawing.

The high alkalinity of concrete normally results in the formation of a passive oxide layer on the surface of steel reinforcement. ${ }^{2,3,9}$ This passivity breaks down in one of two cases: (1) in the case of carbonation, when reduced pore solution alkalinity causes systematic breakdown, ${ }^{10,11}$ or (2) when the chloride concentration is sufficient to cause localized breakdown. ${ }^{12-17}$ In the case of chloride-induced corrosion, breakdown of the passivity of embedded steel occurs at some threshold level of chloride ion concentration. ${ }^{13}$ This critical concentration $C^{*}$ depends mainly on the cement content of the concrete mixture and its alkali reserves, ${ }^{13}$ among many other parameters. ${ }^{14} \mathrm{~A}$ wide range has been reported for $\mathrm{C}^{*}{ }^{15} \mathrm{Absent}$ any significant $\mathrm{pH}$ reduction, many sources suggest $C^{*}$ near $0.4 \%$ by mass of cement, while others suggest that the concept of a critical threshold level to predict corrosion initiation is itself flawed. ${ }^{15}$

Major external sources of chlorides in normal concrete include deicing salts or brines (for concrete in colder climates) and seawater (for concrete in marine environments). In both cases, chlorides intrude through the exposed surface into the concrete. Migration of ionic contaminants through concrete can occur by several mechanisms, including diffusion, absorption, and permeation. ${ }^{18}$ The latter two occur when a fluid laden with ions either flows through the pore structure of concrete or intrudes into the concrete by capillary action. The former is driven by a concentration gradient according to Fick's second law, $\frac{\partial C}{\partial t}=D \Delta C$, where $D$ is the diffusivity, $C$ is the concentration of the ion of interest, $t$ is time, and $\Delta=\nabla^{2}=\left\{\frac{\partial^{2}}{\partial x_{1}^{2}}+\cdots+\frac{\partial^{2}}{\partial x_{n}^{2}}\right\}$ is the Laplacian operator.

It should be noted that, since concrete is heterogeneous, the diffusivity $D$ is more accurately referred to as the apparent diffusivity as determined experimentally. A small diffusivity results in slower transport of ions. The diffusivity decreases with the so-called quality of concrete, which is another way of saying that the same properties that influence mechanical strength also influence the diffusivity. The apparent chloride diffusivity of concrete depends mainly on the pore structure - the porosity, pore connectivity, and tortuosity - and for ordinary portland cement, concrete is normally in the range of $10^{-12} \mathrm{~m}^{2} / \mathrm{s} \leq D \leq 10^{-10} \mathrm{~m}^{2} / \mathrm{s} .{ }^{19-21}$ Some sources have reported diffusivities as low as $10^{-14} \mathrm{~m}^{2} / \mathrm{s}$ for concretes made with supplementary cementitious materials. ${ }^{19}$

Typical approaches to prevent or delay corrosion in steel-reinforced concrete include those that modify the reinforcing material, those that modify the concrete material, or those that modify the concrete section. Steel reinforcement can be protected with an impermeable coating (e.g., epoxy ${ }^{22-25}$ or zinc $^{22,26,27}$ ) or replaced with a noncorroding alternative (e.g., stainless steel ${ }^{28,29}$ or fiber-reinforced polymer ${ }^{30-32}$ ). The concrete materials can be specified to limit the background chloride concentration, and the diffusivity of the concrete can be improved to slow chloride transport through it. ${ }^{2,33}$ Finally, the concrete section can be modified to provide increased cover thickness and further delay the onset of corrosion. Advanced approaches like cathodic protection are also available. ${ }^{34-36}$

Limiting the background chloride concentration effectively increases the amount of chlorides that must penetrate the concrete to initiate corrosion. The maximum allowable chloride concentration of aggregates for use in structural concrete is determined from ACI 318, Building Code Requirements for Structural Concrete, which limits the water-soluble chloride ion concentration by weight of cement, as shown in Table 1. ${ }^{37}$ The building code provides stricter limits for prestressed concrete, where the design is far more sensitive to section loss in the reinforcing steel. Limits range from 0.06 to $1.00 \%$ by mass of cement. Meanwhile, the chloride threshold for corrosion has been reported in the range $0.17-0.25 \%{ }^{13}$ The allowable maximum concentration in Table 1 considers the sum contribution from all mixture components. The allowable chloride concentration in the aggregate therefore depends on the cement content and chloride concentration of the cement and mix water and may be further limited when chloride-based admixtures are used. ASTM C1602, Standard 
TABLE 1

Maximum allowable water-soluble chloride concentration in concrete ${ }^{37}$

\begin{tabular}{lcc}
\hline & $\begin{array}{r}\text { Allowable Maximum Chloride Concentration, } \\
\text { \% by Weight of Cement }\end{array}$ \\
\cline { 2 - 3 } Exposure Class & 1.00 & Prestressed Concrete \\
\hline C0: Dry or protected from moisture & 0.30 & 0.06 \\
C1: Exposed to moisture but not chlorides & 0.15 & 0.06 \\
C2: Exposed to moisture and chlorides & 0.06 \\
\hline
\end{tabular}

Specification for Mixing Water Used in the Production of Hydraulic Cement Concrete, dictates the maximum chloride concentration in mixing water as $500 \mathrm{ppm}$ for prestressed concrete or reinforced concrete bridge decks and $1,000 \mathrm{ppm}$ for other reinforced concrete, ${ }^{38}$ while ACI 318 simply states that mixing water "shall not contain deleterious amounts of chloride ion." ${ }^{\text {37 }}$ Meanwhile, neither ACI 318 nor the relevant ASTM standard ASTM C150, Standard Specification for Portland Cement ${ }^{39}$ dictate the allowable chloride content in portland cement.

The effects of salt contaminants in concrete are varied and include degradation of the fresh, mechanical, and durability properties as well as an increased susceptibility to corrosion of embedded steel (these effects are well summarized by a recent review on this topic ${ }^{40}$ ). Nevertheless, there will be situations when the desire will arise to ignore best practices and to use off-spec aggregates that are contaminated by salt. A few likely examples include marine aggregates and reclaimed aggregates. It may be attractive to use marine aggregates in areas where highquality aggregates are in short supply. Seawater normally has a total salinity near $3.5 \%$ and contains a number of salts (most notably sodium and magnesium chlorides, which respectively contribute about 78 and $15 \%$ of the total salinity). ${ }^{41}$ Aggregates that encounter seawater or salt spray in their natural condition or during storage or transport are therefore likely to contain a relatively high concentration of chlorides and other salts. Meanwhile, efforts to improve sustainability have inspired research into the beneficial reuse of reclaimed concrete aggregate (RCA) and reclaimed asphalt pavement (RAP) as aggregate in new concrete. A recent joint study between Utah State University and University of Konstanz revealed that concern over potential contamination of reclaimed aggregates is a primary barrier to their adoption within the United States. ${ }^{42}$ These concerns are echoed in the literature. ${ }^{43,44}$ The contaminants likely to be present in RCA or RAP include those to which concrete is likely to be exposed in service, e.g., sulfates, alkali-reactive minerals, and chlorides.

Because of the potential contribution of salts from contaminated aggregates to corrosion of reinforcing steel, it will be useful to understand their fate and transport within the concrete matrix. This article first shows that existing 1-D solutions of Fick's law used to describe chloride transport in concrete are ill-conditioned to model the problem of salt-contaminated aggregates in concrete. It then introduces two 3-D solutions that are better suited to describe the problem. The authors demonstrate manipulation of these solutions to assess how the sphere of influence within which a salt-contaminated aggregate may contribute to corrosion initiation in steel-reinforced concrete. Finally, the authors acknowledge several weaknesses of the analysis and discuss how they can be overcome through future work.

This article is an early step in developing a fundamental understanding of the mobility of salts from contaminated aggregates in concrete. The knowledge developed herein will help answer questions related to the suitability for use in concrete of reclaimed aggregates that are potentially contaminated with salts or virgin aggregates from marine climates that are contaminated with seawater or salt spray. The analysis presented here is also broadly applicable to describe the mobility of any ionic contaminant that may be present within concrete or aggregates.

\section{1-D Diffusion}

The problem of 1-D diffusion from a continuous source in a semi-infinite medium is familiar to most concrete professionals. This is the problem used to approximate the penetration of chloride ions into concrete from external sources. This is also the fundamental basis for the determination of the apparent chloride diffusivity $D$ from 
the salt ponding test. ${ }^{45-47}$ The problem considers a continuous source, meaning that the source concentration is constant with time. This is true for the salt ponding test, wherein the surface concentration is kept constant under rigorous experimental control. Similarly, it is true for concrete that is continually exposed to seawater. It is less true for concrete that is only occasionally exposed to seawater or deicing salts. In any case, diffusion occurs in a single dimension $z$, which is the depth below some surface that is exposed to chloride ions.

$\mathrm{Crank}^{48}$ gives the concentration gradient $C(z, t)$ resulting from a continuous source concentration $C_{0}(t)=C_{0}$ in a semi-infinite medium with diffusivity $D$ as follows:

$$
\frac{C(z, t)}{C_{s}}=\operatorname{erfc}\left(\frac{z}{\sqrt{4 D t}}\right)=1-\operatorname{erf}\left(\frac{z}{\sqrt{4 D t}}\right)
$$

where $C_{s}$ is the concentration at the exposed surface, which, incidentally, is not equivalent to the source concentration $C_{0}$. This is illustrated in figure 1 for $D t \in\left\{10^{-3}, 10^{0}, 10^{3}\right\}$. Figure 2 shows contour plots of the same cases. The first and third cases show the general behavior as $D t \rightarrow 0$ or $D t \rightarrow \infty$, respectively. It can easily be seen that, since the source is continuous, the equilibrium condition is the uniform concentration $C_{s}$ :

$$
\lim _{t \rightarrow \infty} C(z, t)=C_{s}
$$

FIG. 1

Normalized

concentration gradients

for 1-D diffusion from a

continuous source in a

semi-infinite medium.
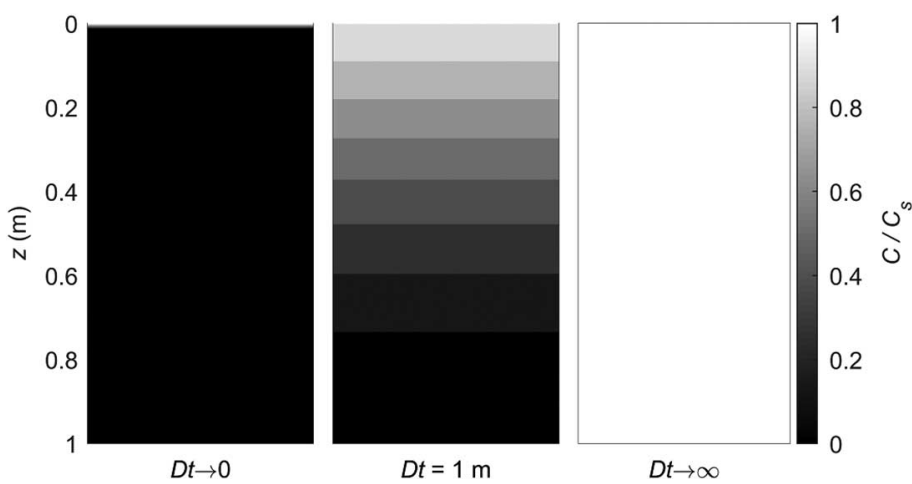

FIG. 2

Contour plots of normalized

concentration gradients for 1-D diffusion from a continuous source in a semi-infinite medium.

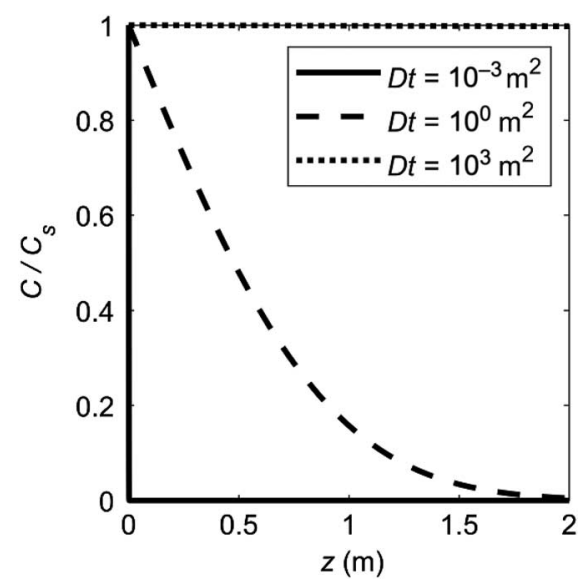


In this and following discussions, the authors present figures without units. This is done for convenience so that the various effects can be shown more generally. The reader will recall that the diffusivity $D$ has units of area per time, and the concentration has units of mass per volume. If the units of length, mass, and time are meters, grams, and seconds, respectively, the units of concentration and diffusivity are parts per million $\left(\mathrm{g} / \mathrm{m}^{3}\right)$ and $\mathrm{m}^{2} / \mathrm{s}$, respectively. Diffusivities of concrete are normally $10^{-10} \mathrm{~m}^{2} / \mathrm{s}$ or lower. The quantity $D t$ has units of area and can be taken as an indication of the timescale relative to the diffusivity.

Concentration gradients of the kind presented in figures 1 and 2 are generally representative of those observed for in-service concrete ${ }^{49-51}$ and ponding tests, ${ }^{45}$ although discrepancies are often noted near the exposed surface. ${ }^{52,53}$

The problem of 1-D diffusion in concrete has been discussed at length throughout the literature (e.g., Thomas and Bamforth, ${ }^{19}$ Luping and Gulikers, ${ }^{21}$ McGrath and Hooton, ${ }^{45}$ and Snyder ${ }^{54}$ ), so the preceding discussion is in some ways superfluous. However, its inclusion draws attention to several fundamental differences between the classic problem of diffusion of chloride ions in concrete and the new problem of diffusion of chlorides from salt-contaminated aggregates. The first difference relates to the location of the source. The 1-D problem considers an external source: seawater exposure, deicing salts and brines, or other sources that penetrate through the concrete surface. Meanwhile, salt-contaminated aggregates represent an internal source. This reveals the second difference in the two problems, which is the dimensionality of the problem. Since external sources penetrate through the concrete surface, diffusion occurs in one dimension $(C=C(z))$. An internal source diffuses in three dimensions $(C=C(x, y, z)$ in Cartesian systems or $C(r, \theta, \phi)$ in spherical systems, where the latter often reduces to $C(r)$ ). Finally, the 1-D problem considers a continuous source-one whose concentration does not degrade with time and thus theoretically provides an infinite mass of diffusing material. Meanwhile, salt-contaminated aggregates contain a finite mass of salt and thus represent an instantaneous source-one whose concentration is time dependent. Although equation (1) gives an accurate description of the salt ponding problem, it is ill-conditioned to describe the mobility of chlorides from salt-contaminated aggregates. The following sections introduce two models of increasing complexity that are better conditioned to handle this emerging problem.

\section{3-D Diffusion}

The following discussions demonstrate 3-D diffusion models for application to the problem of diffusive transport of salts from contaminated aggregates. The assumption that the diffusivity is not directionally dependent, i.e., $D_{x}=D_{y}=D_{z}=D$, allows for the mathematical treatment of a single spatial variable $r$, the radial distance from the source, where $r^{2}=x^{2}+y^{2}+z^{2}$.

\section{3-D DIFFUSION FROM AN INSTANTANEOUS POINT SOURCE IN AN INFINITE MEDIUM}

Consider an instantaneous point source-an infinitesimal slug of diffusing material. The instantaneous point source contains a finite mass $M$ of diffusing material within a volume $V=0$; the initial concentration is therefore $C_{0}=\infty$. Crank ${ }^{48}$ gives the concentration $C$ at time $t$ and location $r$ as the following:

$$
C(r, t)=\frac{M}{(4 \pi D t)^{3 / 2}} \cdot e^{-\frac{r^{2}}{4 D t}}
$$

The concentration at the point source is then as follows:

$$
C_{\max }(t)=C(r=0, t)=\frac{M}{(4 \pi D t)^{3 / 2}}
$$

Then:

$$
C(r, t)=C_{\max }(t) e^{-\frac{r^{2}}{4 D t}}
$$


The equilibrium condition $C_{\infty}$ is solved by taking the limit of equation (3) as $t \rightarrow \infty$ :

$$
C_{\infty}=\lim _{t \rightarrow \infty} C(r, t)=0
$$

This condition arises from the consideration of a finite source in an infinite medium. The true equilibrium condition, considering some finite volume $V$ within which the point source diffuses, is $C_{\infty}=M / V$. Nevertheless, this is in stark contrast to the equilibrium condition in the 1-D problem with a continuous source, which converges to the uniform concentration $C_{0}$.

Figure 3 shows the relative concentration $C / M$ for 3-D diffusion from an instantaneous point source in an infinite medium as a function of the distance $r$ from the source for various $D t$. The concentration is constant near the source and then decreases sharply at some distance $r$ from the source. The distance from the source at which the concentration decreases varies with the time scale $D t$ and ranges from $r=10^{-2}$ for $D t=10^{-3}$ to $r=10^{4}$ for $D t=10^{3}$. In the latter case, the concentration in the constant region is very low $\left(C / M<10^{-5}\right)$. One of the major problems with this model is that when $D t$ is very small, this distance may be much smaller than the typical diameter of an aggregate. Thus, the model predicts zero concentration in an area that is known to be occupied by a salt-contaminated aggregate.

Figure 4 shows contour plots of the same concentration fields shown in figure 3 . The concentration field predicted by the 3-D point source model and shown in both figures is starkly different from that predicted by the 1-D model (fig. 1), highlighting the importance of introducing the 3-D diffusion models.

It is also interesting to look at the maximum concentration $C_{\max }=C(r=0, t)$ as defined by equation (4). Figure 5 shows the normalized maximum concentration $C_{\max } / M$ as a function of the time scale $D t$ for 3-D diffusion from an instantaneous point source. The maximum concentration shows a logarithmic decrease with time, from $C_{\max } / M>10^{10}$ when $D t=10^{-10}$ to $C_{\max } / M<10^{-10}$ when $D t=10^{10}$. As discussed above, the model predicts that $C_{\max } \rightarrow 0$ as $D t \rightarrow \infty$ because it assumes an infinite medium. In practice, the system approaches the equilibrium concentration $C_{\infty}=M / V$.

The point source model is attractive for the problem of salt-contaminated aggregates mainly because of its relative mathematical simplicity. Aggregates do have volume, and to model them as infinitesimal slugs of diffusing material is in many ways problematic, especially given the assumption of infinite initial concentration that follows. Fick's law states that the diffusion flux is proportional to the concentration gradient (i.e., $\partial C / \partial t=D \Delta C$ ). The predicted diffusion flux is greatly accelerated under the assumption of infinite initial concentration, and the point source model will therefore overestimate both the rate of diffusion of salts from contaminated aggregates and the concentration of salts at any given time. The point source model may underestimate the concentration

FIG. 3

Normalized

concentration gradients

for 3-D diffusion from an

instantaneous point

source in an infinite

medium.

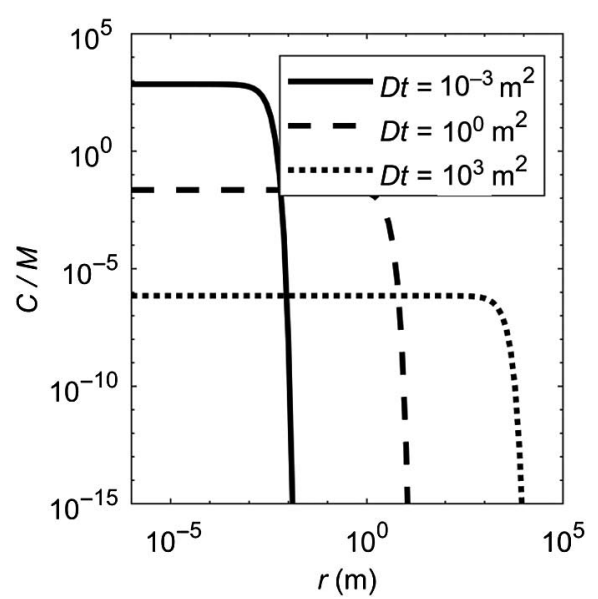


FIG. 4

Contour plots of normalized

concentration gradients for 3-D diffusion from an instantaneous point source in an infinite medium (source at $\left.r=0 ; r^{2}=x^{2}+y^{2}+z^{2}\right)$.

(A) $D t=10^{-3} \mathrm{~m}^{2}$;

(B) $D t=10^{\circ} \mathrm{m}^{2}$;

(C) $D t=10^{3} \mathrm{~m}^{2}$.

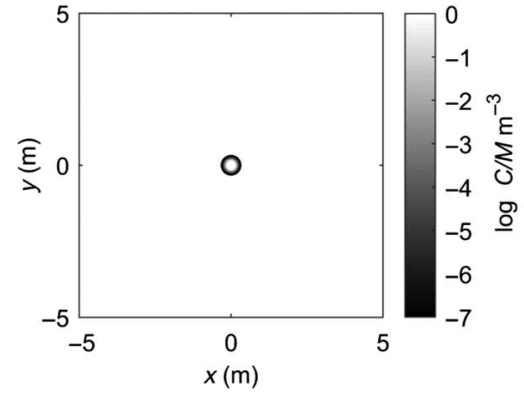

(A)

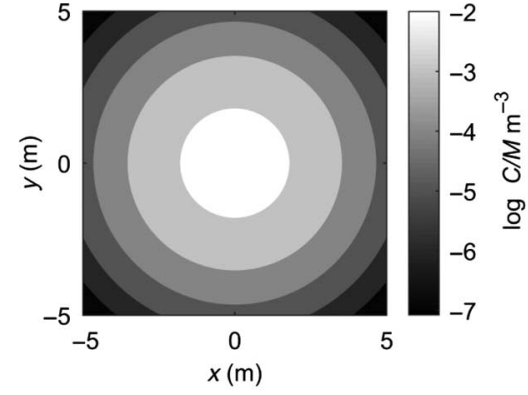

(B)

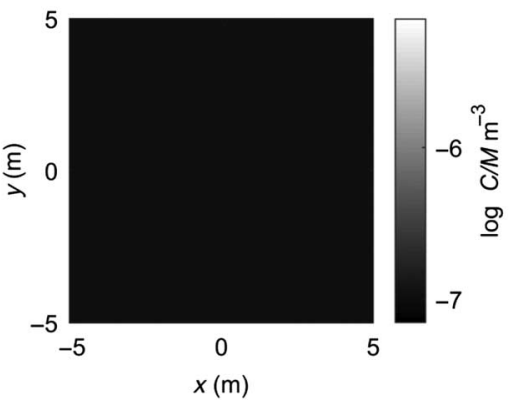

(C)

FIG. 5

Maximum concentration for 3-D diffusion from an instantaneous point source in an infinite medium.

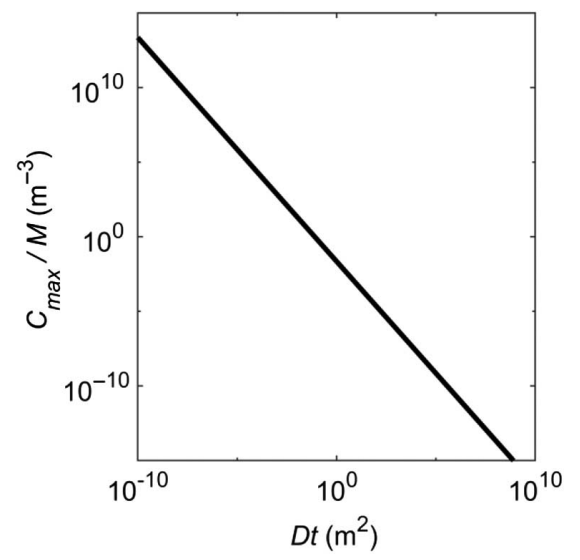

depending on the location relative to the assumed aggregate radius. Consideration of a volumetric source, as in the following section, offers a more accurate representation of the problem at the expense of an increase in mathematical complexity.

\section{3-D DIFFUSION FROM AN INSTANTANEOUS SPHERICAL SOURCE IN AN INFINITE MEDIUM}

The previous discussion is predicated on the assumption that an aggregate with a finite volume and finite concentration of salt can be effectively modeled as an infinitesimal source of infinite concentration. Consider instead 
FIG. 6

Normalized

concentration gradients for 3-D diffusion from an instantaneous spherical source in an infinite medium.

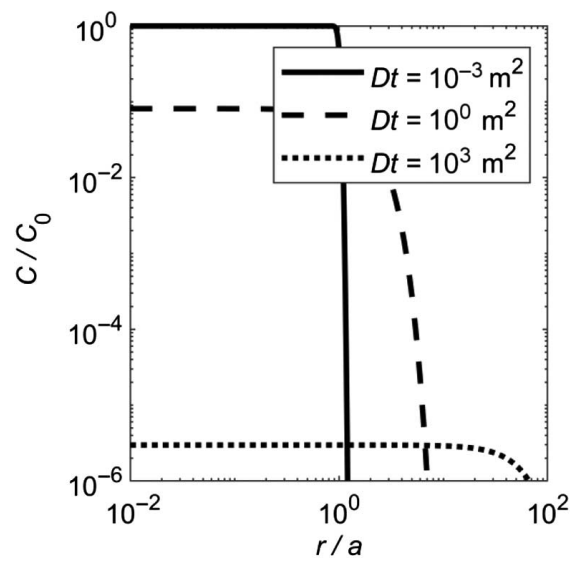

an instantaneous spherical source-a sphere of radius $a$ and volume $V$ with a finite and uniform concentration $C_{0}$ of diffusing material. Crank $^{48}$ gives the concentration field $C(r, t)$ as follows*:

$$
C(r, t)=\frac{1}{2} C_{0}\left(\operatorname{erf} \frac{a+r}{2 \sqrt{D t}}+\operatorname{erf} \frac{a-r}{2 \sqrt{D t}}\right)-\frac{C_{0}}{r} \sqrt{\frac{D t}{\pi}}\left[e^{-\frac{(a-r) 2}{4 D t}}-e^{-\frac{(a+r) 2}{4 D t}}\right]
$$

This can be rewritten more succinctly as the following:

$$
\frac{C(r, t)}{C_{0}}=\frac{\operatorname{erf} \alpha(r, t)}{2}+\frac{\operatorname{erf} \beta(r, t)}{2}-\sqrt{\frac{D t}{\pi r^{2}}}\left(e^{-\beta^{2}(r, t)}-e^{-\alpha^{2}(r, t)}\right)
$$

where:

$$
\alpha(r, t)=\frac{a+r}{2 \sqrt{D t}} \quad \beta(r, t)=\frac{a-r}{2 \sqrt{D t}}
$$

The highest concentration to which the cementitious matrix or embedded steel may come in contact is at the surface of the aggregate. Recognizing that:

$$
\lim _{r \rightarrow a} \alpha(r, t)=\frac{a}{\sqrt{D t}} \quad \lim _{r \rightarrow a} \beta(r, t)=0
$$

and that $\operatorname{erf}(0)=0$, the surface concentration $C_{s}(t)=C(r=a, t)$ is as follows:

$$
\frac{C_{s}(t)}{C_{0}}=\frac{\operatorname{erf} \frac{a}{\sqrt{D t}}}{2}-\sqrt{\frac{D t}{\pi a^{2}}} e-\frac{a^{2}}{D t}
$$

The initial concentration $C_{0}$ is the quotient of the mass of diffusing material and the volume of the spherical particle. Equations (7), (8), and (11) can thus be written in terms of the mass $M$ :

$$
M=\frac{4 \pi a^{3}}{3} C_{0} \quad C_{0}=\frac{3 M}{4 \pi a^{3}}
$$

Figure 6 shows the relative concentration $C / C_{0}$ for an instantaneous spherical source in an instantaneous medium as a function of the relative distance $r / a$ from the centroid of the source and the product of time and *Many copies of Crank $^{48}$ erroneously list the numerator of the second error function term as $a+r$ instead of $a-r$. 
diffusivity $D t$. The concentration remains uniform inside the source $(r / a \leq 1)$, regardless of $D t$. When $D t$ is small, having the practical meaning that the time scale is small relative to the diffusivity, the concentration degrades quickly near the surface of the source particle. Conversely, when $D t$ is large, the uniform concentration extends a great distance into the medium. Contour plots of the same relationships are shown in figure 7.

Figure 8 shows that the surface concentration of the spherical source increases with the source radius $a$ and is inversely related to the square root of time and diffusivity $\sqrt{D t}$. For relatively large source radii or at small time scales, i.e., $a / \sqrt{D t} \geq 1$, the surface concentration approaches the initial concentration $C_{0}$. Conversely, for small source radii or large time scales relative to the diffusivity $(a / \sqrt{D t} \leq 1)$, the surface concentration decreases

FIG. 7

Contour plots of normalized

concentration gradients for 3-D diffusion from an instantaneous spherical source in an infinite medium (source at $r=0 ; r^{2}=x^{2}+y^{2}+z^{2}$.

(A) $D t=10^{-3} \mathrm{~m}^{2}$;

(B) $D t=10^{\circ} \mathrm{m}^{2}$;

(C) $D t=10^{3} \mathrm{~m}^{2}$.

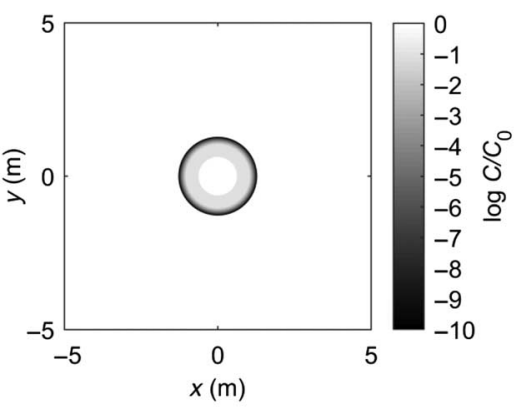

(A)

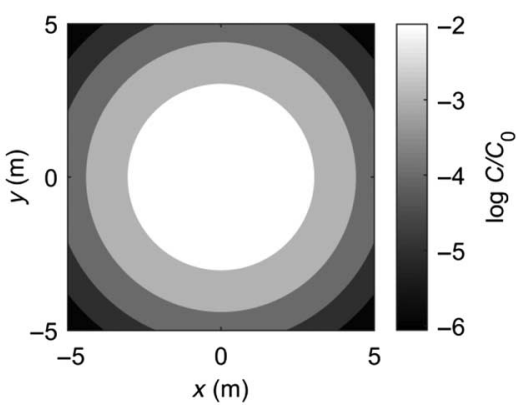

(B)

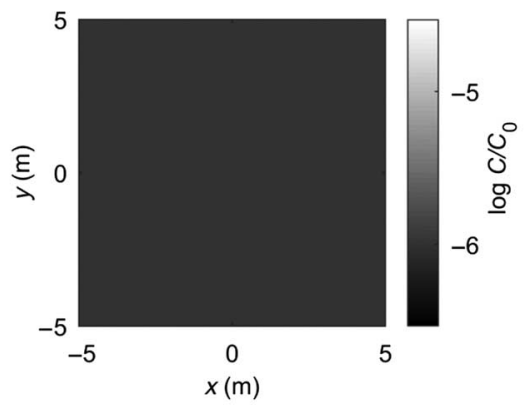

(C)

\section{FIG. 8}

Surface concentration $C_{s}=C(r=a)$ for $3-D$ diffusion from an instantaneous spherical source in an infinite medium.

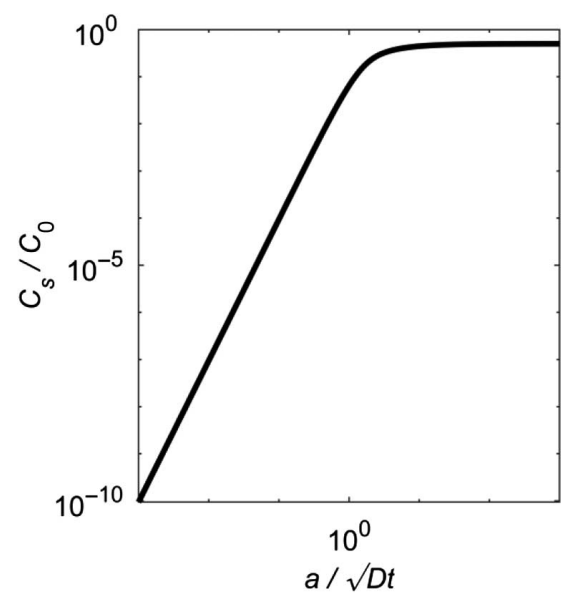


exponentially. The practical implication is that the concentration of salts at the surface of contaminated aggregates will degrade much more quickly for smaller aggregates. This stands to reason, as the surface area increases relative to the mass of salt for smaller particle sizes.

It also stands to reason that the spherical source model should converge to the point source model as the source radius decreases (i.e., as $a \rightarrow 0$ ). This can be shown mathematically by noting equation (11) and the definition of the error function:

$$
\operatorname{erf} z \equiv \frac{2}{\sqrt{\pi}} \int_{0}^{z} e^{-t^{2}} \mathrm{~d} t
$$

This solution is not trivial, and it is more convenient to show convergence graphically. Figure 9 shows the concentration gradients for the point source model and the spherical source model with decreasing $a$ for a system with unit mass, diffusivity, and time. The figure illustrates that, as the size of the spherical source decreases, the spherical source model approaches the point source model. The figure shows the effect of decreasing size with constant mass, but the effect is the same as the initial concentration increases $\left(C_{0} \rightarrow \infty\right)$. The practical implication is that, while the point source model is in many ways deficient, its suitability for modeling the transport of salts from contaminated aggregates improves for smaller aggregates or those with higher initial salt concentrations. The error increases with $a^{2}$ or with the volume of the spherical source. At worst, the error in figure 9 is $0.15 \%$ when $a=0.1$ but increases to $15 \%$ when $a=1$. The error may be several orders of magnitude when $a$ is large or when $C_{0}$ is small. Note that references here to small or large $a$ and $C_{0}$ are relative to $D$ or $t$.

The spherical source model is an improvement over the point source model, particularly for larger or more weakly contaminated aggregates. However, it still requires experimental validation to assess its ability to model the transport of salts from contaminated aggregates. There are also a few remaining problems with the underlying assumptions. These will be discussed later in the manuscript.

\section{AFFECTED RADIUS}

The initiation of corrosion in steel-reinforced concrete depends on some chloride concentration threshold. It is therefore convenient to define the affected radius or volume-the region around a single salt-contaminated aggregate within which the concentration exceeds that critical value. If $C^{\star}$ is the critical concentration, the affected radius is $r^{\star}=r:\left\{C\left(r \leq r^{\star}\right) \geq C^{\star}\right\}$. This is illustrated in figure 10 .

In this context, the concept of affected radius is analogous to the idea of a protected paste region in internally cured concrete. The protected paste region ${ }^{55,56}$ describes the region around a saturated lightweight aggregate within which the degree of saturation is enough to mitigate self-desiccation. The affected volume similarly
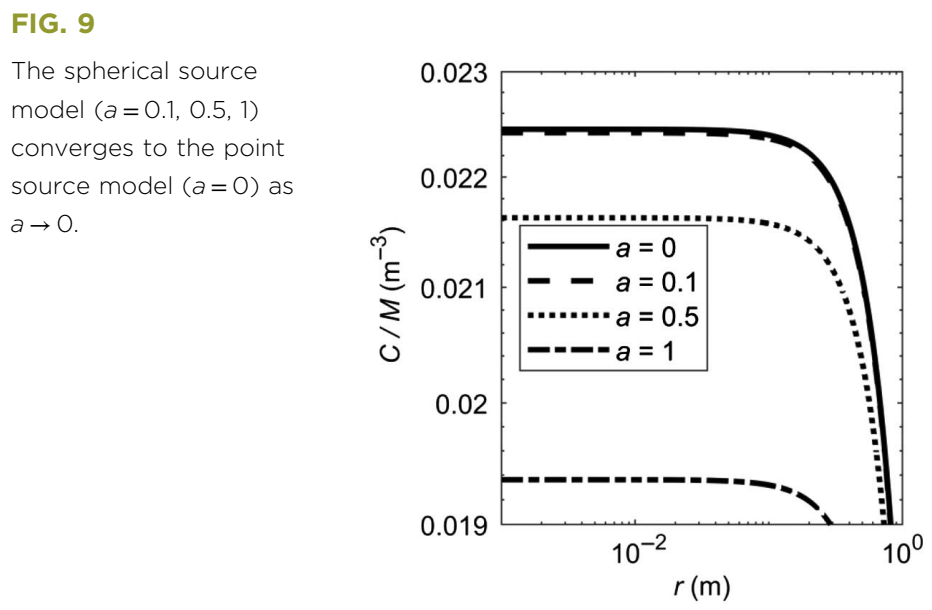
FIG. 10

Affected radius

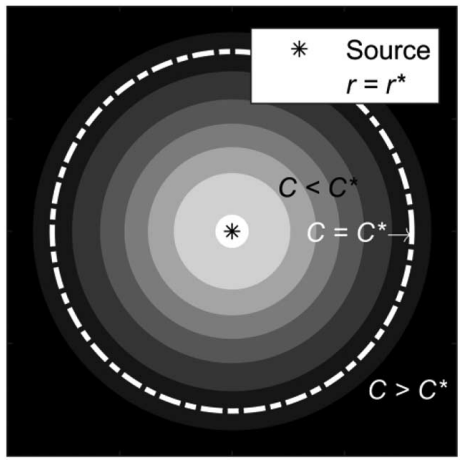

describes the region around an aggregate within which the concentration of chloride is enough to initiate breakdown of the passivity of reinforcing steel and subsequent corrosion. It should be noted before moving on from this analogy that, while a large protected paste region is desirable because it is indicative of a higher level of protection against autogenous shrinkage, a low affected volume is desirable in the case of salt-contaminated aggregates because it suggests a lesser chance of corrosion problems.

Computing the affected radius also has broad experimental applications. Several authors have demonstrated the colorimetric indication of chloride penetration depth in concrete using silver nitrate on its own or in conjunction with fluorescein or potassium chromate. ${ }^{57-63}$ Each of these colorimetric indicators change color when exposed to chloride above some critical concentration. These methods are typically used for qualitative analysis of the comparative diffusivity of concrete mixtures; those that experience a higher depth of penetration, as indicated by color change, have a higher apparent diffusivity. However, by understanding the relationship between source concentration, critical concentration, and affected radius, these colorimetric indicators can be used to infer the diffusivity of concrete (or other media) without extensive titration analyses. The development of the following equations will therefore be of great utility in developing 3-D diffusion experiments in future work.

Solutions for the affected radii for the cases of instantaneous point and spherical sources in infinite media are given as follows. These are solved from the concentration fields taken from Crank. ${ }^{48}$ While Crank provides the concentration fields, he does not solve for or discuss affected radii. In fact, a thorough review of literature citing Crank does not reveal any such solutions or references to the idea of an affected radius. This is not to say that any great advancement has been made in the present work, but rather to point out that this concept may be new and is certainly worthy of consideration for the present application.

The mathematical formulation for the concentration field caused by 3-D diffusion from an instantaneous point source in an infinite medium is given by equation (3). The affected radius for a point source is found by solving for $r$ :

$$
r^{*}\left(C^{*}, t\right)=\sqrt{-4 D t \cdot \ln \left[\frac{C^{*}}{M} \cdot(4 \pi D t) \frac{3}{2}\right]}=\sqrt{-4 D t \cdot \ln \frac{C^{*}}{C_{\max }(t)}}
$$

Figure 11 shows the affected radius as a function of $D t$ for various critical concentrations relative to the mass $C^{\star} / M$. The affected radius increases with $\log (D t)$ up to some maximum as the substance diffuses further into the medium. The affected radius reaches some maximum value, after which it decreases rapidly to zero as the degree of dilution brings the concentration below the critical value. The maximum affected radius can be determined by differentiating equation (14) and solving for $D t: \partial r^{\star} / \partial D t=0$. Similarly, the value of $D t$ at which $r^{\star} \rightarrow 0$ could be 
FIG. 11

Affected radius for instantaneous point source in an infinite medium.

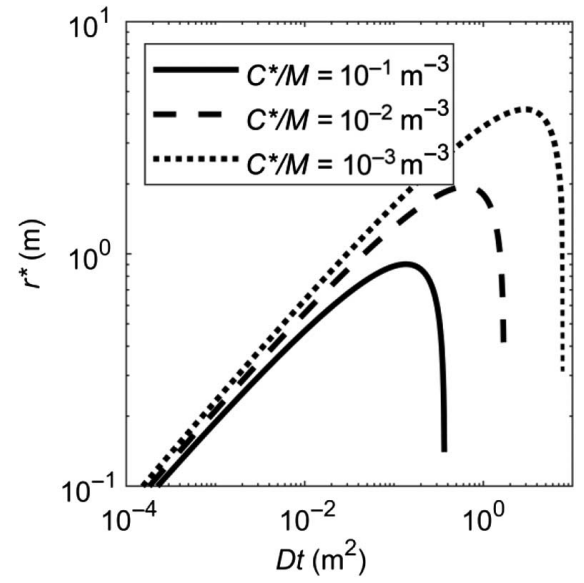

found algebraically. Both depend only on $C^{\star} / M$. These calculations are not presented here for the sake of brevity, but some graphical observations are useful. In the most conservative case, where $C^{\star}=1,000 M$, the affected radius reaches a maximum of about 5 for $D t$ of the order $10^{0}$ and goes to 0 as $D t \rightarrow 10^{1}$. As $C^{\star}$ increases relative to $M$, both the maximum affected radius and the time at which it occurs are reduced. The physical meaning of $C^{\star} / M$ is not completely intuitive because of the point source assumption, but an example is useful. Consider a spherical aggregate of diameter $2 a$ and initial concentration $C^{0}$ that is approximated as a point source. The mass is $M=\frac{4 \pi a^{3}}{3} C_{0}$ or, conversely, the initial concentration is $C_{0}=\frac{3 M}{4 \pi a^{3}}$. For a $10-\mathrm{mm}$ diameter spherical aggregate, $C^{\star}=1,000 M$ is approximately the same as $C_{0}=2 C^{\star}$, or the contamination level of the aggregate, by mass, is twice the critical concentration $C^{\star}$, by mass of concrete.

Similarly, the mathematical formulation for the concentration field caused by 3-D diffusion from an instantaneous spherical source in an infinite medium is given by equation (7). The complexity of the spherical source model—with its error function and exponential terms on $r$-makes its solution for $r$ inconvenient. It is, however, trivial to solve for the affected radius numerically. ${ }^{\dagger}$ Thus computed, figure 12 shows the affected radius normalized by the source radius $r^{\star} / a$ and for various critical concentrations relative to the initial concentration $C^{\star} / C^{0}$. For the most conservative case, where $C_{0}=1,000 C^{\star}$, the affected radius reaches a maximum of about $r_{\max }^{*} \approx 7 a$ for $D t \approx 10^{1}$ and decreases to 0 quickly thereafter. Volume increases with the radius cubed, meaning that in this conservative case, the maximum region within which the concentration exceeds the critical value is nearly 350 times the initial volume of the source. The practical implication is that when the salt concentration of a contaminated aggregate is very large relative to the chloride threshold level, each aggregate can render an extremely large region of the concrete susceptible to corrosion. However, this scenario is extremely unlikely in practice and would almost certainly result in instantaneous initiation of corrosion. The maximum affected radius is only about 2 when $C_{0}=10 C^{\star}$. In practice, this means that the affected volume is only about 8 times the initial aggregate volume when the salt concentration in the aggregate is 10 times the chloride threshold level (for example, if the chloride threshold $C^{\star}$ is $0.01 \%$ by mass of concrete and the aggregate contamination level is $0.1 \%$ by mass).

Figure 13 shows contour plots of affected radii for point and spherical sources. Figure $13 A$ shows the affected radius $r^{\star}$ for a point source as a function of $C^{\star} / M$ and $D t$. Figure 13B shows the normalized affected radius $r^{\star} / a$ for a spherical source as a function of $C^{\star} / C_{0}$ and $D t$. Both show the general trend of affected radius, which decreases with critical concentration and increases with time until some maximum, after which it decreases 
FIG. 12

Affected radius for instantaneous spherical source in an infinite medium.

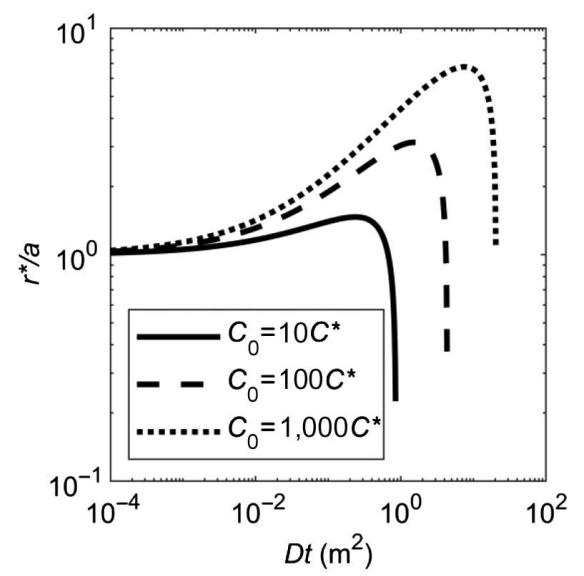

FIG. 13

Contour plot of affected radius for instantaneous sources in an infinite medium: ( $A$ ) point source; $(B)$ spherical source.

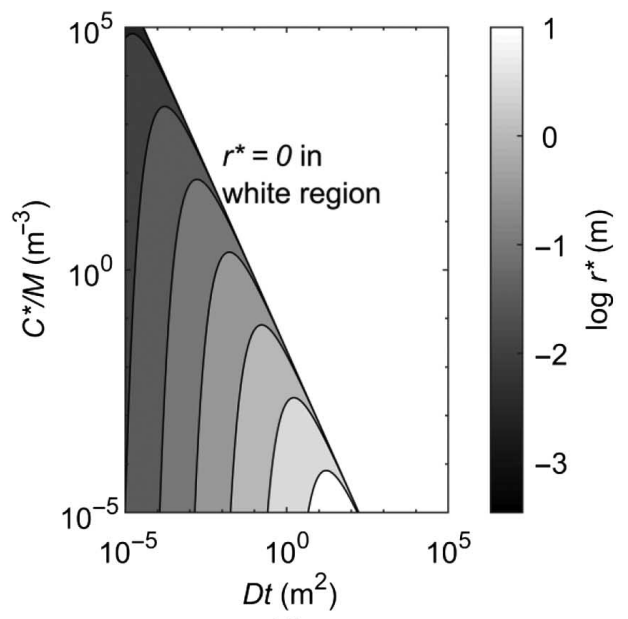

(A)

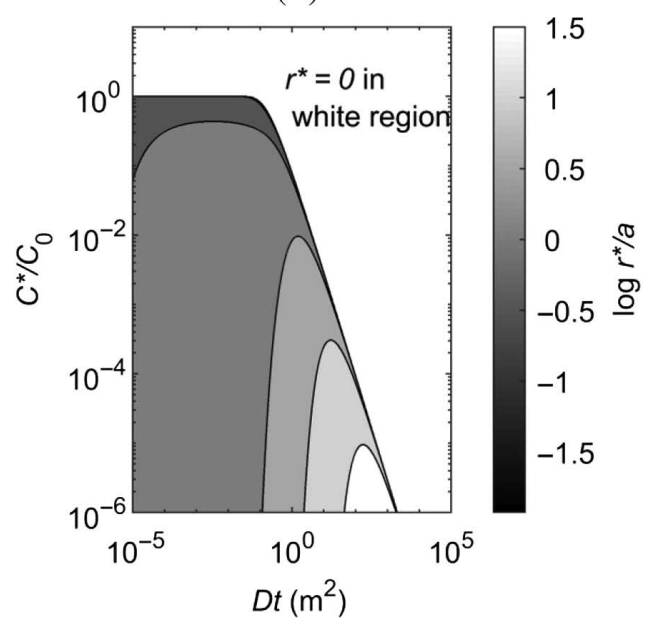

(B) 
rapidly to zero. For the spherical source, the affected radius is naturally zero when $C^{\star}>C_{0}$. No such zero region is observed for the point source because the infinitesimal assumption forces normalization by the mass and not the initial concentration. These figures show that the affected radius can be very large $\left(r^{\star}>10 a\right)$ when the level of salt contamination in the aggregate is very high relative to the threshold level for corrosion. However, when the concentration of the aggregate is at or near the threshold level, the region of influence is confined to the aggregate itself $\left(r^{\star} \leq a\right)$. The reader will note that $r^{\star} \leq a$ has the practical meaning that the only place where the concentration exceeds the threshold level is inside the aggregate itself.

It should further be noted that, aside from the differences in normalization, the only practical difference between figure $13 A$ and $13 B$ is that the affected radius goes to zero at smaller $D t$ for the point source than for the spherical source. This results from the acceleration of diffusion under the point source model, as discussed previously in "3-D Diffusion." The assumption that the point source is infinitesimal means that the prediction for the affected radius will start out much smaller but reach its maximum more quickly. In general, the affected radius for the spherical source will be larger than that for the point source. As with the concentration gradients shown in figure 9, the affected radii for the spherical source model approach the point source solution as $a \rightarrow 0$ or as $C_{0} \rightarrow \infty$, although this is not shown here. In practice, this means that the simpler point source model is more accurate for smaller or more highly concentrated aggregates.

\section{TIME-DEPENDENT DIFFUSIVITY}

The preceding discussion makes several problematic assumptions. One of the most glaring of these is that the analyses consider a constant diffusivity. The diffusivity in hardened concrete is known to decrease approximately logarithmically with time. ${ }^{19}$ At first blush, this seems trivial: $D$ becomes $D(t)$ in equations (3) and (7), and the computations become slightly more complex. However, this is mathematically incorrect because it does not satisfy Fick's second law, $\partial C / \partial t=D \Delta C$.

$\mathrm{Crank}^{48}$ summarizes the solution process for a time-dependent diffusivity $D=D(t)$. Consider the differential equation:

$$
\mathrm{d} T=D(t) \mathrm{d} t
$$

Then, Fick's second law becomes the following:

$$
\frac{\partial C}{\partial T}=\Delta C
$$

As expected, equation (15) is equivalent to Fick's law when $D$ is constant.

\section{MULTIPLE SOURCES}

Another deficit in the point and spherical source analyses is that they consider only a single source in an infinite medium. Concrete made with salt-contaminated aggregates includes potentially millions of sources per cubic meter. These vary in size and shape but are approximately uniform in initial concentration and follow a somewhat regular spacing.

Superposition (fig. 14) is a simple, albeit fundamentally flawed way to model the concentration fields for multiple salt-contaminated aggregates. The matrix of aggregates discussed previously presents a network of opposing concentration gradients. Fick states that the diffusion flux is proportional to the concentration gradient, so these are opposing concentration gradients. Superposition does not consider the effect of these opposing concentration gradients. The result is that superposition will underestimate the time required to approach the equilibrium condition (i.e., at $t \rightarrow \infty$, the concentration necessarily approaches some constant value $C_{\infty}=M_{\text {tot }} / V_{\text {concrete, }}$ where $M_{\text {tot }}$ is the total mass of diffusing material (salt) in all particles, and $V_{\text {concrete }}$ is the total volume of concrete). In practice, this will underestimate the concentration near the particles and overestimate it remotely from the particles. That superposition is so fundamentally ill-conditioned that to model diffusion from multiple sources will likely mean that the inaccuracy will outweigh the simplicity. An analytical 
FIG. 14 Superposition to model concentration gradients from multiple sources (not to scale).
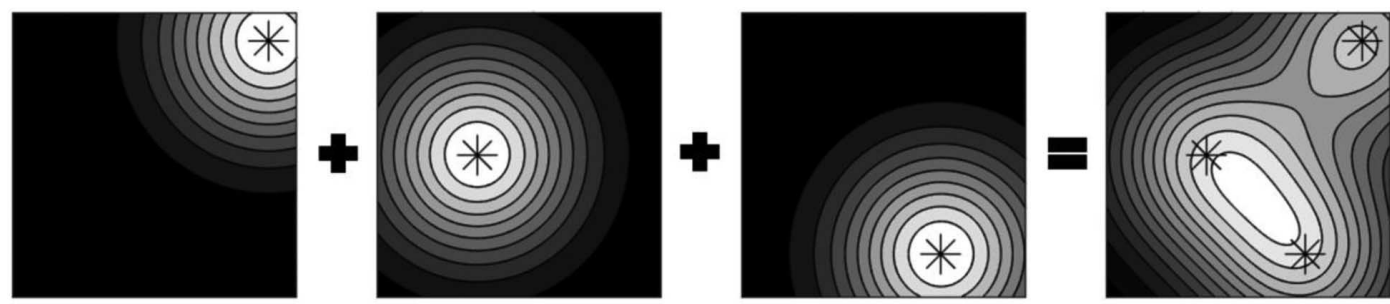

solution that will consider the effects of transient opposing concentration gradients is preferred. The authors are continuing to work toward this goal.

\section{ADDITIONAL PROBLEMS WITH THE ANALYSIS}

Previous sections elaborated on the mathematical treatment for consideration of time-dependent diffusivity, but the diffusivity is also dependent on moisture content. This is most obvious for fresh concrete, where the very high moisture content means that the diffusivity of salts in the mixture is extremely high. The analytical model does not consider the effects of chloride binding, nor the presence of chlorides within the mortar matrix. Both of these will affect the concentration gradient that drives diffusion.

As previously discussed, the point source model considers only an infinitesimal mass of salt. This assumption is acceptable for very small aggregates wherein the initial concentration is large, but the accuracy of the model suffers as the aggregate size increases. This is problematic because the aggregate size is not likely uniform; this shortcoming also affects the utility of the spherical source model. The spherical source model further assumes a uniform initial concentration. In practice, salts are expected to be more concentrated at the surface of the contaminated aggregates. It is also unrealistic to assume that aggregates are of a uniform shape, although the spherical assumption is likely acceptable given specified limitations on aspect ratio.

The analysis assumes that all salts present in the aggregates are free and not chemically bound. It also fails to consider that some salts that diffuse from the contaminated aggregate may become bound within the concrete matrix. Some chlorides become chemically bound to reaction products within the cement paste matrix. ${ }^{64-66}$ These bound chlorides should not be considered part of the transport problem. Similarly, some chlorides within the aggregates are likely to be bound, so the initial aggregate concentration should be considered as the watersoluble (free) chloride content rather than the acid-soluble (total) concentration. The analysis can easily be modified such that the initial concentration $C_{0}$ is the free salt concentration in the aggregate. However, it is not clear whether bound salts within the concrete matrix fully contribute to the concentration gradient that drives further diffusion.

Furthermore, it is possible and often convenient to model diffusion problems using the finite element method. It is unknown if the proposed analytical analysis provides any better information than a simple sensitivity analysis using the finite element method. Future work will compare results from analytical and finite element analyses to answer this question. Future work will also seek to determine the impact of the point source assumption on the accuracy of the predicted concentration field, helping to determine whether the improvement in accuracy outweighs the increased mathematical complexity of the spherical source model.

\section{Summary and Conclusions}

Typically, discussions of diffusion in concrete relate to the intrusion of salts into concrete from external sources. Thus, existing analytical descriptions of diffusion in concrete consider 1-D diffusion from a continuous source. This 
solution is ill-conditioned to describe the diffusion of salts from internal sources like contaminated aggregates, which are a finite source and diffuse in three dimensions. This article explores two 3-D models that offer a more realistic description of the problem. The first is for 3-D diffusion from an instantaneous point source in an infinite medium and models a salt-contaminated aggregate as an infinitesimal slug with a finite mass of salt. The second is for 3-D diffusion from an instantaneous spherical source in an infinite medium, which models a salt-contaminated aggregate as a spherical solid with a uniform and finite concentration of salt. The point source model provides a more mathematically simple description of the problem at the cost of inaccuracy resulting from the assumption that the aggregate source is infinitesimal. Meanwhile, the spherical source model provides a more realistic description of the physical problem, but the mathematics are more complex. Both models are limited in that (1) they only model diffusion from a single source rather than multiple sources at a semiregular interval, and (2) they consider a constant diffusivity rather than one that depends on age of concrete or moisture content.

The article introduces equations to describe the concentration fields in both the point source and spherical source problems, as given by $\mathrm{Crank}^{48}$ in 1979. The authors then introduce the concept of and solve for the affected radius, which describes the region around a salt-contaminated aggregate within which the salt concentration exceeds some critical value. Since the initiation of corrosion in steel-reinforced concrete depends on exceeding some chloride concentration threshold, this is of great utility. Wherever possible, the authors draw attention to major flaws in the underlying assumptions for these models, including the consideration of a constant diffusivity and a single source in an infinite medium. The article presents mathematical foundations for revising the analysis to consider multiple sources and a time-dependent diffusivity. Despite these and other faults in the assumptions, this work represents a first step toward an analytical description of the mobility of salts from contaminated aggregates in concrete. Future work will seek to (1) address the limitations described above and (2) validate the model using experimental evidence and numerical simulations.

The major remaining question is what does this mean for concrete made with salt-contaminated aggregates? That question demands a discussion at least as long as this and, for the sake of brevity, will be answered elsewhere.

\section{References}

1. G. H. Koch, M. P. Brongers, N. G. Thompson, Y. P. Virmani, and J. H. Payer, Corrosion Cost and Preventive Strategies in the United States (McLean, VA: Federal Highway Administration, 2002).

2. S. Ahmad, "Reinforcement Corrosion in Concrete Structures, its Monitoring and Service Life Prediction--A Review," Cement and Concrete Composites 25, nos. 4-5 (May-July 2003): 459-471, https://doi.org/10.1016/S0958-9465(02)00086-0

3. N. Berke, A. Bentur, and S. Diamond, Steel Corrosion in Concrete: Fundamentals and Civil Engineering Practice (Boca Raton, FL: CRC Press, 2014).

4. D. Li, R. Wei, L. Li, X. Guan, and X. Mi, "Pitting Corrosion of Reinforcing Steel Bars in Chloride Contaminated Concrete," Construction and Building Materials 199 (February 2019): 359-368, https://doi.org/10.1016/j.conbuildmat.2018.12.003

5. P. Chindaprasirt and W. Chalee, "Effect of Sodium Hydroxide Concentration on Chloride Penetration and Steel Corrosion of Fly Ash-Based Geopolymer Concrete under Marine Site," Construction and Building Materials 63 (July 2014): 303-310, https://doi.org/10.1016/j.conbuildmat.2014.04.010

6. W. Zhu, R. François, Q. Fang, and D. Zhang, "Influence of Long-Term Chloride Diffusion in Concrete and the Resulting Corrosion of Reinforcement on the Serviceability of RC Beams," Cement and Concrete Composites 71 (August 2016): 144152, https://doi.org/10.1016/j.cemconcomp.2016.05.003

7. D. Chen and S. Mahadevan, "Chloride-Induced Reinforcement Corrosion and Concrete Cracking Simulation," Cement and Concrete Composites 30, no. 3 (March 2008): 227-238, https://doi.org/10.1016/j.cemconcomp.2006.10.007

8. R. Zhang, A. Castel, and R. François, "Concrete Cover Cracking with Reinforcement Corrosion of RC Beam during Chloride-Induced Corrosion Process," Cement and Concrete Research 40, no. 3 (March 2010): 415-425, https://doi. org/10.1016/j.cemconres.2009.09.026

9. J. P. Broomfield, Corrosion of Steel in Concrete: Understanding, Investigation and Repair (Boca Raton, FL: CRC Press, 2003).

10. J. A. Gonzalez, J. S. Algaba, and C. Andrade, "Corrosion of Reinforcing Bars in Carbonated Concrete," British Corrosion Journal 15, no. 3 (July 2013): 135-139, https://doi.org/10.1179/bcj.1980.15.3.135

11. M. Moreno, W. Morris, M. G. Alvarez, and G. S. Duffó, "Corrosion of Reinforcing Steel in Simulated Concrete Pore Solutions: Effect of Carbonation and Chloride Content," Corrosion Science 46, no. 11 (November 2004): 2681-2699, https://doi.org/10.1016/j.corsci.2004.03.013 
12. L. Li and A. A. Sagüés, "Chloride Corrosion Threshold of Reinforcing Steel in Alkaline Solutions-Open-Circuit Immersion Tests," Corrosion 57, no. 1 (January 2001): 19-28, https://doi.org/10.5006/1.3290325

13. G. K. Glass and N. R. Buenfeld, "The Presentation of the Chloride Threshold Level for Corrosion of Steel in Concrete," Corrosion Science 39, no. 5 (May 1997): 1001-1013, https://doi.org/10.1016/S0010-938X(97)00009-7

14. R. B. Figueira, A. Sadovski, A. P. Melo, and E. V. Pereira, "Chloride Threshold Value to Initiate Reinforcement Corrosion in Simulated Concrete Pore Solutions: The Influence of Surface Finishing and pH," Construction and Building Materials 141 (June 2017): 183-200, https://doi.org/10.1016/j.conbuildmat.2017.03.004

15. U. Angst, B. Elsener, C. K. Larsen, and Ø. Vennesland, "Critical Chloride Content in Reinforced Concrete-A Review," Cement and Concrete Research 39, no. 12 (December 2009): 1122-1138, https://doi.org/10.1016/j.cemconres.2009.08.006

16. A. Costa and J. Appleton, "Chloride Penetration into Concrete in Marine Environment-Part I: Main Parameters Affecting Chloride Penetration,” Materials and Structures 32, no. 4 (May 1999): 252, https://doi.org/10.1007/BF02479594

17. M. F. Montemor, A. M. P. Simões, and M. G. S. Ferreira, "Chloride-Induced Corrosion on Reinforcing Steel: From the Fundamentals to the Monitoring Techniques," Cement and Concrete Composites 25, nos. 4-5 (May-July 2003): 491-502, https://doi.org/10.1016/S0958-9465(02)00089-6

18. L. Basheer, J. Kropp, and D. J. Cleland, "Assessment of the Durability of Concrete from Its Permeation Properties: A Review," Construction and Building Materials 15, nos. 2-3 (March/April 2001): 93-103, https://doi.org/10.1016/S09500618(00)00058-1

19. M. D. A. Thomas and P. B. Bamforth, "Modelling Chloride Diffusion in Concrete: Effect of Fly Ash and Slag," Cement and Concrete Research 29, no. 4 (April 1999): 487-495, https://doi.org/10.1016/S0008-8846(98)00192-6

20. P. Lehner, P. Ghosh, and P. Konečný, "Statistical Analysis of Time Dependent Variation of Diffusion Coefficient for Various Binary and Ternary Based Concrete Mixtures," Construction and Building Materials 183 (September 2018): 75-87, https://doi.org/10.1016/j.conbuildmat.2018.06.048

21. T. Luping and J. Gulikers, "On the Mathematics of Time-Dependent Apparent Chloride Diffusion Coefficient in Concrete," Cement and Concrete Research 37, no. 4 (April 2007): 589-595, https://doi.org/10.1016/j.cemconres.2007.01.006

22. S. Dong, B. Zhao, C. Lin, R. Du, R. Hu, and G. X. Zhang, "Corrosion Behavior of Epoxy/Zinc Duplex Coated Rebar Embedded in Concrete in Ocean Environment," Construction and Building Materials 28, no. 1 (March 2012): $72-$ 78, https://doi.org/10.1016/j.conbuildmat.2011.08.026

23. A. A. Sagüés, J. B. Lee, X. Chang, H. Pickering, E. Nystrom, W. Carpenter, S. C. Kranc, et al., Corrosion of Epoxy-Coated Rebar in Florida Bridges (Tallahassee, FL: Florida Department of Transportation, 1994).

24. J. L. Smith and Y. P. Virmani, Performance of Epoxy Coated Rebars in Bridge Decks (McLean, VA: Federal Highway Administration, 1996).

25. L. L. Smith, R. J. Kessler, and R. G. Powers, “Corrosion of Epoxy-Coated Rebar in a Marine Environment," Transportation Research Circular 403 (March 1993): 36-45.

26. O. T. de Rincón, O. Pérez, E. Paredes, Y. Caldera, C. Urdaneta, and I. Sandoval, "Long-Term Performance of ZnO as a Rebar Corrosion Inhibitor," Cement and Concrete Composites 24, no. 1 (October 2002): 79-87, https://doi.org/10.1016/ S0958-9465(01)00029-4

27. J. A. González and C. Andrade, "Effect of Carbonation, Chlorides and Relative Ambient Humidity on the Corrosion of Galvanized Rebars Embedded in Concrete," British Corrosion Journal 17, no. 1 (January 2013): 21-28, https://doi.org/10. $1179 / 000705982798274589$

28. P. Castro-Borges, O. Troconis-Rincón, E. I. Moreno, A. A. Torres-Acosta, M. Martinez-Madrid, and A. Knudsen, "Performance of a 60-Year-Old Concrete Pier with Stainless Steel Reinforcement," Materials Performance 41, no. 10 (October 2002): 50-55.

29. A. Knudsen, F. M. Jensen, O. Klinghoffer, and T. Skovsgaard, "Cost-Effective Enhancement of Durability of Concrete Structures by Intelligent Use of Stainless Steel Reinforcement," in Proceedings of the International Conference on Corrosion and Rehabilitation of Reinforced Concrete Structures (Washington, DC: Federal Highway Administration, 1998).

30. R. Okelo and R. L. Yuan, "Bond Strength of Fiber Reinforced Polymer Rebars in Normal Strength Concrete," Journal of Composites for Construction 9, no. 3 (June 2005): 203-213, https://doi.org/10.1061/(ASCE)1090-0268(2005)9:3(203)

31. C. E. Bakis, L. C. Bank, V. L. Brown, E. Cosenza, J. F. Davalos, J. J. Lesko, A. Machida, S. H. Rizkalla, and T. C. Triantafillou, "Fiber-Reinforced Polymer Composites for Construction-State-of-the-Art Review," Journal of Composites for Construction 6, no. 2 (May 2002): 73-87, https://doi.org/10.1061/(ASCE)1090-0268(2002)6:2(73)

32. B. Benmokrane, P. Wang, T. M. Ton-That, H. Rahman, and J.-F. Robert, "Durability of Glass Fiber-Reinforced Polymer Reinforcing Bars in Concrete Environment," Journal of Composites for Construction 6, no. 3 (August 2002): 143-153, https://doi.org/10.1061/(ASCE)1090-0268(2002)6:3(143)

33. M. K. Kassir and M. Ghosn, "Chloride-Induced Corrosion of Reinforced Concrete Bridge Decks," Cement and Concrete Research 32, no. 1 (January 2002): 139-143, https://doi.org/10.1016/S0008-8846(01)00644-5

34. P. Pedeferri, "Cathodic Protection and Cathodic Prevention," Construction and Building Materials 10, no. 5 (July 1996): 391-402, https://doi.org/10.1016/0950-0618(95)00017-8

35. L. Bertolini, F. Bolzoni, A. Cigada, T. Pastore, and P. Pedeferri, "Cathodic Protection of New and Old Reinforced Concrete Structures," Corrosion Science 35, nos. 5-8 (1993): 1633-1639, https://doi.org/10.1016/0010-938X(93)90393-U

36. K. G. C. Berkeley and S. Pathmanaban, Cathodic Protection of Reinforcement Steel in Concrete (Kent, UK: Butterworths and Company, 1990). 
37. Building Code Requirements for Structural Concrete (ACI 318-14) and Commentary, ACI 318R-14 (Farmington Hills, MI: American Concrete Institute, 2014).

38. Standard Specification for Mixing Water Used in the Production of Hydraulic Cement Concrete, ASTM C1602-12 (West Conshohocken, PA: ASTM International, 2012). https://doi.org/10.1520/C1602_C1602M-18

39 Standard Specification for Portland Cement, ASTM C150-18 (West Conshohocken PA: ASTM International, 2018). https://doi.org/10.1520/C0150_C0150M-18

40. J. Xiao, C. Qiang, A. Nanni, and K. Zhang, "Use of Sea-Sand and Seawater in Concrete Construction: Current Status and Future Opportunities," Construction and Building Materials 155 (November 2017): 1101-1111, https://doi.org/10.1016/j. conbuildmat.2017.08.130

41. A. Neville, "Seawater in the Mixture," Concrete International 23, no. 1 (January 2001): 48-51.

42. D. Palzer, "Entwicklungsstand von Recyclingbeton in Europa und den USA sowie dessen Verbreitung und Förderung in der Baupraxis," (Master's thesis, Hochschule Konstanz University of Applied Sciences, 2016).

43. A. Rao, K. N. Jha, and S. Misra, "Use of Aggregates from Recycled Construction and Demolition Waste in Concrete," Resources, Conservation and Recycling 50, no. 1 (March 2007): 71-81, https://doi.org/10.1016/j.resconrec.2006.05.010

44. W. V. Srubar III, "Stochastic Service-Life Modeling of Chloride-Induced Corrosion in Recycled-Aggregate Concrete," Cement and Concrete Composites 55 (January 2015): 103-111, https://doi.org/10.1016/j.cemconcomp.2014.09.003

45. P. F. McGrath and R. D. Hooton, "Re-Evaluation of the AASHTO T259 90-Day Salt Ponding Test," Cement and Concrete Research 29, no. 8 (August 1999): 1239-1248, https://doi.org/10.1016/S0008-8846(99)00058-7

46. Standard Method of Test for Resistance of Concrete to Chloride Ion Penetration, AASHTO T259 (Washington, DC: American Association of State Highway Transportation Officials, 2002).

47. Standard Test Method for Determining the Penetration of Chloride Ion into Concrete by Ponding, ASTM C1543-10a (West Conshohocken, PA: ASTM International, 2010). https://doi.org/10.1520/C1543-10A

48. J. Crank, The Mathematics of Diffusion (Oxford: Oxford University Press, 1979).

49. K. De Weerdt, D. Orsáková, A. C. A. Müller, C. K. Larsen, B. Pedersen, and M. R. Geiker, "Towards the Understanding of Chloride Profiles in Marine Exposed Concrete, Impact of Leaching and Moisture Content," Construction and Building Materials 120 (September 2016): 418-431, https://doi.org/10.1016/j.conbuildmat.2016.05.069

50. J. Kim, W. J. McCarter, B. Suryanto, S. Nanukuttan, P. A. M. Basheer, and T. M. Chrisp, "Chloride Ingress into Marine Exposed Concrete: A Comparison of Empirical-and Physically-Based Models," Cement and Concrete Composites 72 (September 2016): 133-145, https://doi.org/10.1016/j.cemconcomp.2016.06.002

51. S.-W. Pack, M.-S. Jung, H.-W. Song, S.-H. Kim, and K. Y. Ann, "Prediction of Time Dependent Chloride Transport in Concrete Structures Exposed to a Marine Environment," Cement and Concrete Research 40, no. 2 (February 2010): 302312, https://doi.org/10.1016/j.cemconres.2009.09.023

52. C. Andrade, J. M. Díez, and C. Alonso, "Mathematical Modeling of a Concrete Surface 'Skin Effect' on Diffusion in Chloride Contaminated Media," Advanced Cement Based Materials 6, no. 2 (August 1997): 39-44, https://doi.org/10. 1016/S1065-7355(97)00002-3

53. K. Y. Ann, J. H. Ahn, and J. S. Ryou, "The Importance of Chloride Content at the Concrete Surface in Assessing the Time to Corrosion of Steel in Concrete Structures," Construction and Building Materials 23, no. 1 (January 2009): 239-245, https://doi.org/10.1016/j.conbuildmat.2007.12.014

54. K. A. Snyder, "The Relationship between the Formation Factor and the Diffusion Coefficient of Porous Materials Saturated with Concentrated Electrolytes: Theoretical and Experimental Considerations," Concrete Science and Engineering 3, no. 12 (December 2001): 216-224.

55. D. P. Bentz, P. Lura, and J. W. Roberts, "Mixture Proportioning for Internal Curing," Concrete International 27, no. 2 (February 2005): 35-40.

56. D. P. Bentz and W. J. Weiss, Internal Curing: A 2010 State-of-the-Art Review (Gaithersburg, MD: National Institute of Standards and Technology, 2011).

57. V. Baroghel-Bouny, P. Belin, M. Maultzsch, and D. Henry, “ $\mathrm{AgNO}_{3}$ Spray Tests: Advantages, Weaknesses, and Various Applications to Quantify Chloride Ingress into Concrete. Part 1: Non-Steady-State Diffusion Tests and Exposure to Natural Conditions," Materials and Structures 40, no. 8 (October 2007): 759, https://doi.org/10.1617/s11527-007-9233-1

58. M. Collepardi, A. Marcialis, and R. Turriziani, "Penetration of Chloride Ions into Cement Pastes and Concretes," Journal of the American Ceramic Society 55, no. 10 (October 1972): 534-535, https://doi.org/10.1111/j.1151-2916.1972.tb13424.x

59. F. He, C. Shi, Q. Yuan, X. An, and B. Tong, "Calculation of Chloride Concentration at Color Change Boundary of AgNO3 Colorimetric Measurement," Cement and Concrete Research 41, no. 11 (November 2011): 1095-1103, https://doi.org/10. 1016/j.cemconres.2011.06.008

60. F. He, C. Shi, Q. Yuan, C. Chen, and K. Zheng, "AgNO 3 -Based Colorimetric Methods for Measurement of Chloride Penetration in Concrete," Construction and Building Materials 26, no. 1 (January 2012): 1-8, https://doi.org/10.1016/ j.conbuildmat.2011.06.003

61. N. Otsuki, S. Nagataki, and K. Nakashita, "Evaluation of the $\mathrm{AgNO}_{3}$ Solution Spray Method for Measurement of Chloride Penetration into Hardened Cementitious Matrix Materials," Construction and Building Materials 7, no. 4 (December 1993): 195-201, https://doi.org/10.1016/0950-0618(93)90002-T

62. C.-C. Yang and C.-H. Liang, "A Modified Colorimetric Method to Determine the Chloride Profile from the Ponding Test," Journal of the Chinese Institute of Engineers 37, no. 4 (July 2013): 419-427, https://doi.org/10.1080/02533839.2013. 799948 
63. Q. Yuan, C. Shi, F. He, G. De Schutter, K. Audenaert, and K. Zheng, "Effect of Hydroxyl Ions on Chloride Penetration Depth Measurement Using the Colorimetric Method," Cement and Concrete Research 38, no. 10 (October 2008): 11771180, https://doi.org/10.1016/j.cemconres.2008.04.003

64. K. De Weerdt, A. Colombo, L. Coppola, H. Justnes, and M. R. Geiker, "Impact of the Associated Cation on Chloride Binding of Portland Cement Paste," Cement and Concrete Research 68 (February 2015): 196-202, https://doi.org/10.1016/ j.cemconres.2014.01.027

65. V. J. Azad, A. R. Erbektas, C. Qiao, O. B. Isgor, and W. J. Weiss, "Relating the Formation Factor and Chloride Binding Parameters to the Apparent Chloride Diffusion Coefficient of Concrete," Journal of Materials in Civil Engineering 31, no. 2 (February 2019): 04018392, https://doi.org/10.1061/(ASCE)MT.1943-5533.0002615

66. T. Luping and L.-O. Nilsson, "Chloride Binding Capacity and Binding Isotherms of OPC Pastes and Mortars," Cement and Concrete Research 23, no. 2 (March 1993): 247-253, https://doi.org/10.1016/0008-8846(93)90089-R 